\title{
Micro/Nanofiber-coupled low-dimensional structures for nanophotonic applications -INVITED
}

\author{
Xin $\mathrm{Guo}^{1,2^{*}}$ \\ ${ }^{1}$ State Key Laboratory of Modern Optical Instrumentation, Zhejiang University, Hangzhou 310027, China \\ ${ }^{2}$ College of Optical Science and Engineering, Zhejiang University, Hangzhou 310027, China
}

\begin{abstract}
Optical micro/nanofiber, with diameter close to the wavelength of the waveguided light, leaves a considerably large fractional evanescent fields for coupling with low-dimensional functional nanostructures. Here I introduce our works on micro/nanofiber-coupled low-dimensional structures for nanophotonic applications including gas sensing and ultrashort pulse measurement.
\end{abstract}

\section{Introduction}

An optical micro/nanofiber (MNF) can guide light with tight optical confinement and strong evanescent waves, making it possible to efficiently couple with functional nanostructures [1-3]. Here I introduce our progress on micro/nanofiber-coupled low-dimensional structures, including metal nanoparticles, metal nanowires and semiconductor microwires, for nanophotonic devices and applications [4-8].

\section{MNF-coupled low-dimensional structures and applications}

\subsection{Coupling of MNF with metal nanoparticles}

Owing to their fascinating optical properties originated from localized surface plasmon resonance, metal nanoparticles are attracting increasing attentions as it is possible to confine light into the deep-subwavelength scale in all three dimensions. By attaching metal nanoparticles to the inner edge of a bent nanofiber, we theoretically show a hybrid structure to reduce the bending loss of a sharply-bent optical nanofiber [4]. Due to the near field coupling between the metal nanoparticles and the nanofiber, a considerable fraction of energy can be confined to the interface of the metal nanoparticles and the optical nanofiber, resulting in a significant reduction of the bending loss in the sharply-bent optical nanofiber. It shows the merits of easy operation, high integration, and a very small bending radius down to $1-\mu \mathrm{m}$ level.

\subsection{Coupling of MNF with micro/nanowires}

Chemically synthesized metal nanowires or semiconductor microwires, with atomically smooth surface and well-developed crystal structure, have favorable properties including ultra-tight optical confinement, large surface-to-volume ratio, and high optical nonlinearity for nanophotonic applications such as sensors and optical correlators.

Via ultracompact near-field interaction, we realize effective coupling by directly contacting optical micro/nanofibers with metal nanowires [5,6]. A photonto-plasmon conversion efficiency higher than $80 \%$ is experimentally realized in a microfiber-coupled $\mathrm{Ag}$ nanowire. Based on highly efficient coupling between optical fibers and Ag nanowires, we have assembled allfiber hybrid photon-plasmon components such as hybrid Mach-Zehnder interferometer and ring resonators [6]. For practical applications, we further demonstrate a nanowire plasmonic sensor for detecting ammonia gas $\left(\mathrm{NH}_{3}\right)$ with detection limit lower than $100 \mathrm{ppm}$ and response time of about $300 \mathrm{~ms}$, which offers a promising route to bridge light from macroscopic fiber systems to microscopic nanowire plasmonics for highly localized and flexible sensing applications [7].

Moreover, by counterpropagating ultrafast pulses in a CdTe microwire evanescently coupled to microfibers at both ends, and converting the temporal profile of the pulses into the transverse second harmonic spatial image, we demonstrate a single-microwire optical correlator for femtosecond pulses at 2- $\mu \mathrm{m}$ wavelength [8]. The measured pulse duration from the microwire-based autocorrelator agrees well with that extracted by the standard auto-correlator. Due to the low-loss transmission window for the CdTe microwire in the mid-infrared region, the microwire-based auto-correlator shows great potential for mid-infrared ultrashort pulse measurement.

\section{Conclusions}

Our results above suggest that the micro/nanofibercoupled low-dimensional structures may offer a versatile platform for nanophotonic devices and applications.

* Corresponding author: guoxin@zju.edu.cn 
This research was supported by the National Key Research and Development Project of China (2018YFB2200404), and the Fundamental Research Funds for the Central Universities.

\section{References}

1. L. M. Tong, R. R. Gattass, J. B. Ashcomv, S. L. He, J. Y. Lou, M. Y. Shen, I. Maxwell, E. Mazur, Nature 426, 816 (2003)

2. L. M. Tong, F. Zi, X. Guo, J. Y. Lou, Opt. Commun. 285, 4641 (2012)

3. L. M. Tong, Sensors 18, 903 (2018)

4. Y. X. Yang, J. Gong, Y. X. Gao, H. Wu, X. Guo, P. Wang, L. M. Tong (to be submitted)

5. X. Guo, M. Qiu, J. M. Bao, B. J. Wiley, Q. Yang, X. N. Zhang, Y. G. Ma, H. K. Yu, L. M. Tong, Nano Lett. 9, 4515 (2009)

6. X. Y. Li, W. Li, X. Guo, J. Y. Lou, L. M. Tong, Opt. Express 21, 15698 (2013)

7. X. Guo, Y. B. Ying, L. M. Tong, Acc. Chem. Res. 47, $656(2014)$

8. J. B. Zhang, Z. X. Shi, L. Yang, C. G. Xin, Y. H. Li, Y. Kang, L. Z. Wang, J. Gong, X. Guo, L. M. Tong (to be submitted) 